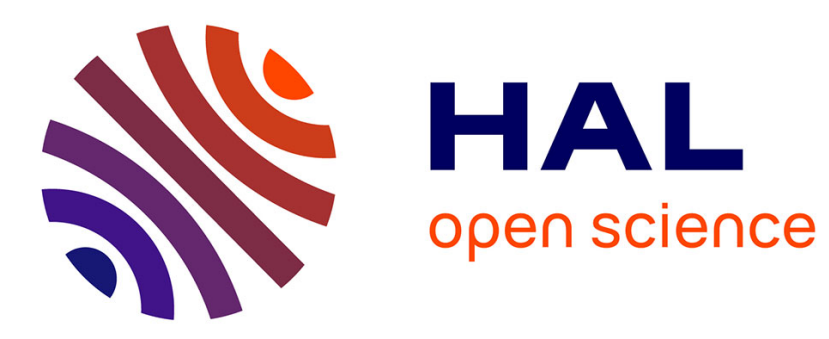

\title{
Heat convection in a vertical channel: Plumes versus turbulent diffusion
}

Mathieu Gibert, Hervé Pabiou, Jean-Christophe Tisserand, Betina B. Gertjerenken, Bernard Castaing, Francesca Chillà

\section{- To cite this version:}

Mathieu Gibert, Hervé Pabiou, Jean-Christophe Tisserand, Betina B. Gertjerenken, Bernard Castaing, et al.. Heat convection in a vertical channel: Plumes versus turbulent diffusion. Physics of Fluids, 2009, 21 (3), pp.035109. 10.1063/1.3085812 . ensl-00377244

\section{HAL Id: ensl-00377244 \\ https://hal-ens-lyon.archives-ouvertes.fr/ensl-00377244}

Submitted on 21 Apr 2009

HAL is a multi-disciplinary open access archive for the deposit and dissemination of scientific research documents, whether they are published or not. The documents may come from teaching and research institutions in France or abroad, or from public or private research centers.
L'archive ouverte pluridisciplinaire HAL, est destinée au dépôt et à la diffusion de documents scientifiques de niveau recherche, publiés ou non, émanant des établissements d'enseignement et de recherche français ou étrangers, des laboratoires publics ou privés. 


\title{
Heat Convection in a Vertical Channel: Plumes versus Turbulent Diffusion
}

\author{
M. Gibert, ${ }^{*}$ H. Pabiou,$^{\dagger}$ J.-C. Tisserand, B. Gertjerenken,$\stackrel{\ddagger}{ }$ B. Castaing, and F. Chillà ${ }^{\S}$ \\ Université de Lyon, ENS Lyon, CNRS \\ 46 Allée d'Italie, 69364 Lyon Cedex 7, France
}

(Dated: December 5, 2008)

\begin{abstract}
Following a previous study (Phys. Rev. Letters 96, 084501, (2006)), convective heat transfer in a vertical channel of moderate dimensions follows purely inertial laws. It would be therefore a good model for convective flows of stars and ocean. Here we report new measurements on this system. We use an intrinsic length in the definition of the characteristic Rayleigh and Reynolds numbers. We explicit the relation between this intrinsic length and the thermal correlation length. Using PIV, we show that the flow undergoes irregular reversals. We measure the average velocity profiles and the Reynolds stress tensor components. The momentum flux toward the vertical walls seems negligible compared to the shear turbulent stress. A mixing length theory seems adequate to describe the horizontal turbulent heat and momentum fluxes, but fails for the vertical ones. We propose a naive model for vertical heat transport inspired by the Knudsen regime in gases.
\end{abstract}

PACS numbers: 47.27

\section{INTRODUCTION}

The Rayleigh-Bénard flow, heat convection between two horizontal plates at different temperatures $T_{h}$ and $T_{c}\left(T_{h}-T_{c}=\Delta T\right)$, has long been the only paradigm for laboratory studies of thermal convection [1-3]. Recent controversies $[2,4-6]$ concerning the occurence of the socalled Kraichnan regime [7] stressed the importance of having a better knowledge of the bulk flow, far from the plates $[8,9]$. However, global quantities such as the Nusselt number $(N u)$ :

$$
N u=\frac{Q H}{\lambda \Delta T}
$$

are mainly controled by the neighborhood of the plates, where the viscosity weakens the convection. The Nusselt number compares the heat flux $Q$ to the purely diffusive one $\lambda \Delta T / H$, where $\lambda$ is the fluid thermal conductivity, and $H$ the vertical distance between plates.

On the other hand, natural convection, such as occuring in stars or planet's atmospheres, is often free of plates. Evaluating heat fluxes generated in such conditions is of prime importance for elucidating the behavior of these systems. In this respect, heat (or mass) convection in a vertical channel $[10-12]$ is closer to these flows. Even if the width $d$ of the channel introduces a characteristic length, the heat flux is everywhere convective, if one exclude the poorly pertinent vertical boundary layers, in contradistinction with the neighborhood of Rayleigh-Bénard plates. Note that a vertical access pit of an underground quarry [13] is another semi-natural example of such a system.

\footnotetext{
*Also at MPI-DS (LFPN) Göttingen.

$\dagger$ Also at Université de Lyon, CNRS.

$¥$ Also at Carl von Ossietzky Universität, Oldenburg

$\S$ Electronic address: Francesca.Chilla@ens-lyon.fr
}

The characteristic non-dimensional numbers have here a slightly different definition. Out of end effects, a constant vertical temperature gradient $\beta=-\partial T / \partial z$ is expected to drive the heat flux, which gives the Nusselt number:

$$
N u=\frac{Q}{\lambda \beta}
$$

One of the control parameters is the Prandtl number $\operatorname{Pr}=\nu / \kappa$, where $\nu$ is the kinematic viscosity, and $\kappa$ the heat diffusivity. The second control parameter is the Rayleigh number:

$$
R a=\frac{g \alpha \beta L^{4}}{\nu \kappa}
$$

where $g$ is the gravitational acceleration, and $\alpha$ the constant pressure thermal expansion coefficient. The choice of the characteristic length $L$ has to be done. It could be taken as $d$, the width of the channel, which a priori appears as the only available length. However, our previous study [12] shows that results are clearer when a different choice is made:

$$
L=a L_{o} \text { with } L_{o}=\frac{\theta}{\beta}
$$

where $\theta$ is a root mean squared temperature fluctuation which will be precised later. In the same study, a characteristic time $\tau_{o}$ was evidenced in the spectrum of temperature fluctuations. Two hypothesis were made for interpreting the data. First, for considering $\theta$ as a temperature, we had to assume that the correlation length is relatively short. Second, we assumed that we could choose the factor $a$ in such a way that $L / \tau_{o}$ be the rms velocity $V_{r m s}$. We thus assumed:

$$
V_{r m s}=\frac{a \theta}{\beta \tau_{o}}
$$

We grossly estimated it from one single PIV experiment. We shall see that the present experiments yield a 
different value for $a$, but confirm that it can be considered as constant. With $L / \tau_{o}$ representing the rms velocity, we can define a Reynolds number:

$$
R e=\frac{L^{2}}{\nu \tau_{o}}
$$

The important point is that this $R e$ is obtained only through thermal measurements, within the same experiments giving $N u$.

The present paper has several purposes. After a detailled presentation of the various experimental techniques and set-ups, we examine the above hypothesis, through a direct measurement of the correlation length. Then we compare the previously [12] measured $N u$ and $R e$ with those obtained with a smaller cell, having a different aspect ratio. A statistical study of the velocity field follows, which allows us to determine a characteristic velocity profile, and the Reynolds stresses. From these Reynolds stresses and their horizontal $(x)$ dependences, a horizontal temperature profile can be determined, as well as the corresponding horizontal heat flux. Before the conclusion, we examine these results from the point of view of the Prandtl mixing length theory.

\section{EXPERIMENTAL SET-UP AND MEASUREMENTS}

We used two different cells, both using water as the fluid. The general set-up is as presented in figure 1 . The vertical channel, in which our measurements are made, connects two chambers, a cold one at its top end and a hot one at its bottom end. Hot plumes are formed on the hot plate. They mix in the hot chamber before to feed a rising, turbulent, irregular flow in the channel. Symetrically, cold plumes are formed on the cold plate, mix in the cold chamber, and feed a sinking flow in the channel. As will be explained below, the walls (excluding the plates), both of the chambers and of the channel, are insulating: no heat flux is entering this way.

The first cell, already described in [12], is mainly constituted of a traditional rectangular Rayleigh-Bénard cell of height $40 \mathrm{~cm}$ and section $40 \times 10 \mathrm{~cm}^{2}$. Thanks to a honeycomb structure (made in PMMA [14]), figure 2a, we avoid convection to appear in about $50 \%$ of the RB cell. The other $50 \%$, the convection zone, has in its central part a channel with a $10 \times 10 \mathrm{~cm}^{2}$ cross section area $(d=10 \mathrm{~cm})$ and a height of $20 \mathrm{~cm}$. This channel is our zone of interest. The walls of the RB cell are $25 \mathrm{~mm}$ thick PMMA.

The second cell, figure $2 \mathrm{~b}$, has been specially designed for this experiment. It consists of two axisymetric chambers, of conical shape, connected through a square channel, $20 \mathrm{~cm}$ in length, of $5 \times 5 \mathrm{~cm}^{2}$ inner area. The walls of the channel are made of PMMA, $10 \mathrm{~mm}$ thick.

For both cells, the upper plate is temperature regulated by a regulated water bath. The bottom plate is heated by the Joule effect in 5 resistors, regularly spaced, for the

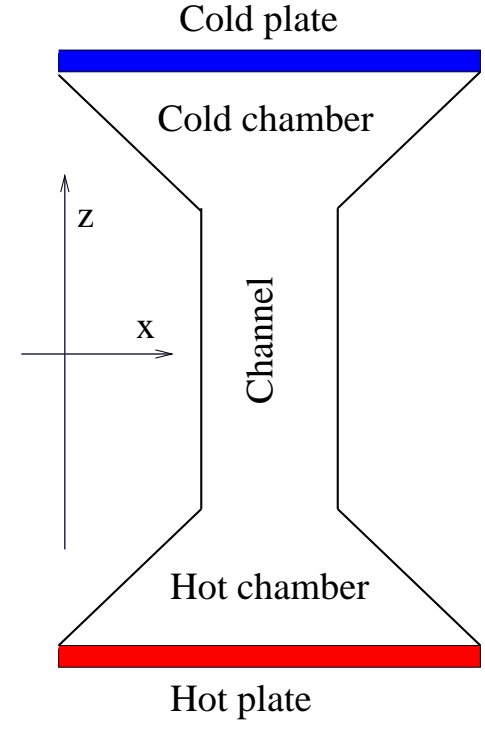

FIG. 1: Schematic view of both cells, clarifying the notations.

first cell, and in a heating wire, forming an Archimede spiral, for the second one. The total power input could go from $3 \mathrm{~W}$ to $300 \mathrm{~W}$ to cover our range of Rayleigh numbers.

For thermal measurements, both cells are thermally insulated. The thermal insulation allows us to limit and control the heat losses in order to precisely measure the heat flux through the cell. Heat losses can occur either via a spurious contact with the hot plate, where the Joule heating power is supplied, or through the vertical walls (or the honeycomb structure in the first cell case). Indeed, the effective heat conductivity of the channel is several thousand times that of water or plastics forming the walls. Heat would have to cross 6 plastic walls for flowing though the honeycomb structure. Looking at shadowgraphs of the first cell, we could verify that no activity, characteristic of convection, occurs in the honeycomb structure.

The second cell is hanged by the cold plate, whose temperature is fixed by a regulating water bath. Then, nothing except air is in contact with the hot plate. We could not hang the first cell the same way. Its hot plate fits into plastic supports. The first cell is further surrounded by a copper screen. This screen is temperature regulated at the average temperature between top and bottom plates, which is maintained constant on a whole set of measurements. In addition, both cells are wrapped in isolating sheets to limit air convection.

As a final thermal characterisation of both cells, we measured the total temperature difference $\Delta T$ between the hot and cold plates, versus the supplied power. It is worth noting that this total temperature difference is the same as for a Rayleigh-Bénard cell having the same plates, and the same distance between them (within 
10\%). The huge constraints imposed here to the bulk flow have little influence on the global Nusselt number. This confirms previous studies [15] showing the poor influence of the bulk flow on the Nusselt number in the Rayleigh-Bénard geometry.

For vertical temperature gradient measurements, we use a bridge made of four resistive thermometers from the same batch [16] located at the corners of a rectangle $5 \mathrm{~cm}(\operatorname{resp} 2.5 \mathrm{~cm})$ wide and of height $h=10 \mathrm{~cm}(\operatorname{resp} 5 \mathrm{~cm})$ for the first (resp second) cell. To maintain this geometry without being too intrusive, we use an "I" structure as shown on figure 2 (b). The resistors are connected as a Wheatstone bridge, in such a way that the two upper (resp. lower) ones are in opposite situation (no common point). As the resistors have very close temperature dependencies, the output of the bridge is poorly sensitive to the average temperature, but highly sensitive to a temperature difference between top and bottom branches of the bridge. The input to the bridge is $30 \mathrm{~Hz}, 0.1 \mathrm{~V}$ voltage from a Agilent 33220A generator. The output is measured by a differential lock-in amplifier Stanford Research SR830 DSP. The sensitivity of the bridge to temperature differences is typically $2 \mathrm{mV} / \mathrm{K}$, and the order of magnitude of the output for zero temperature difference (the offset of the bridge) is $10^{-4} \mathrm{~V}$.

Heat losses have been estimated in situ, looking at the root mean squared amplitude of fluctuations of the bridge output. We extrapolate this rms amplitude to a zero value to establish the corresponding supplied power. Indeed, if the supplied power is less than heat losses, the fluid stratifies which suppresses the turbulent temperature fluctuations. Such an extrapolation also gave the exact output of the bridge for zero temperature gradient in the channel.

For temperature correlations, we used smaller resistive thermometers [17]. Both thermometers are positioned on the same vertical line, each at the bottom end of a " $L$ " structure, the vertical bars being made of two tubes, one sliding inside the other (figure 2a). Both resistances $r_{1}$ and $r_{2}$ are measured through a 2-wire Agilent 34970A multiplexer. Measurements have been taken by blocks of $2 \times 20000$. A complete measurement for a given distance between thermometers lasted two days, for nearly 100 blocks. While converting the $r_{i}$ values in temperature is a priori possible through the calibration we made, the small amplitude of temperature fluctuations makes it useless for correlation studies. We directly consider the correlation of resistance fluctuations (see section III).

Velocity fields are measured through a commercial Particule Imaging Velocimetry (PIV) system [18]. The flow is seeded with hollow glass spheres, $10 \mu \mathrm{m}$ in diameter [19]. Before every set of measurements, we make work the experiment some hours. Spheres with the wrong average density have time to settle at the top or the bottom of the cell. A vertical laser sheet, $2 \mathrm{~mm}$ wide, is obtained from a $1 \mathrm{~W}$ continuous laser [20]. For the first cell, a slot in one of the honeycomb structures allows the free propagation of the sheet.

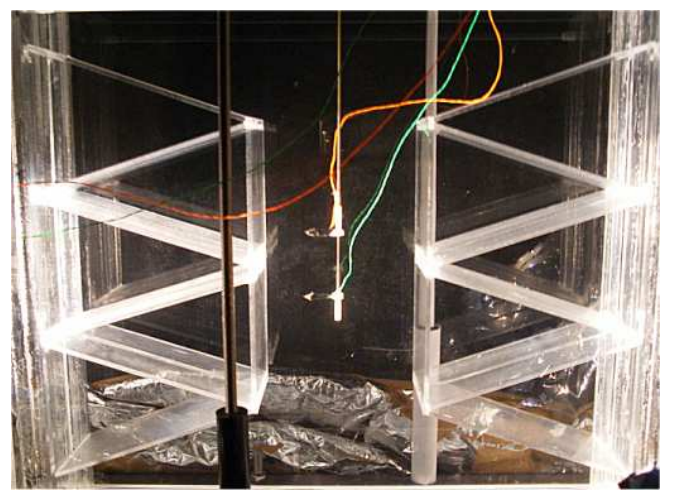

(a)

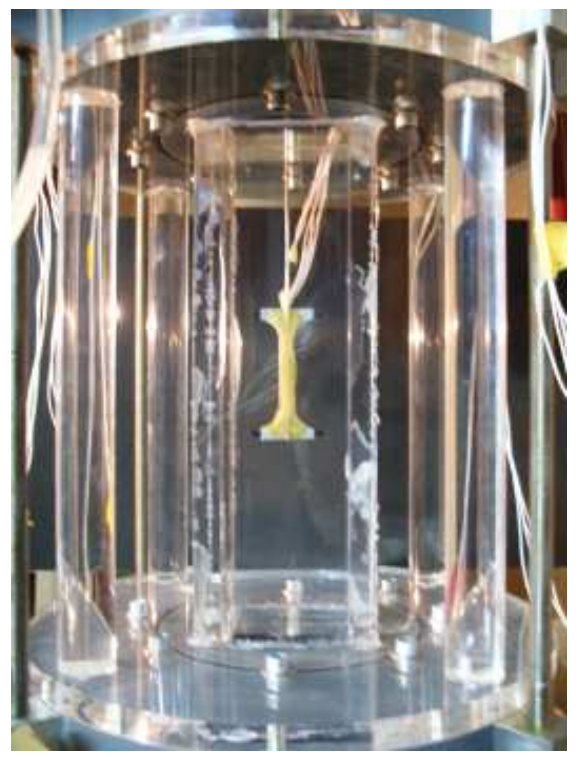

(b)

FIG. 2: (a) Picture of the first cell (without the insulation and the thermal screen). The "L" structures used for temperature correlation measurements are shown in the channel. (b) Picture of the second cell (without the insulation). The short version of the bridge $(5 \mathrm{~cm}$ high $\times 2.5 \mathrm{~cm}$ wide $)$ can be seen in the channel.

We use a $30 \mathrm{~Hz}$ camera [21], $1200 \times 1600$ pixels, 12 bits grey resolution. The buffer is able to store 20 frames. We thus register by blocks of 20 frames, $50 \mathrm{~ms}$ between frames, whose comparison gives 19 two dimensional, two components velocity fields, with $3 \mathrm{~mm}$ resolution. We systematically choose to register 80 such blocks, but with different spacing between blocks. The total registration time goes from 2 to 10 hours. 


\section{CROSS-CORRELATION MEASUREMENTS}

Our goal, in this study of the spatial and temporal thermal correlations is twofold. First we want to check if the correlation length $\ell$ is smaller than the size of the bridge, so that bridge output fluctuations can be interpreted as temperature fluctuations, a fundamental hypothesis of [12]. We want also to check our second hypothesis, namely that $a$ (Eqs. 4,5$)$ is constant and verify its value.

We thus performed, in the first cell, the correlation measurements for two different values of the Rayleigh number, both at the same Prandtl number $\operatorname{Pr}=5.2$, corresponding to an average temperature of the cell of $30^{\circ} \mathrm{C}$ :

- For the high power one, the power input in the bottom plate is $235 \mathrm{~W} . N u=1.37 \times 10^{4}$, and $L_{o}=$ $11.4 \mathrm{~cm}$.

- For the low power one, the power input in the bottom plate is $23 \mathrm{~W} . N u=4.0 \times 10^{3}$, and $L_{o}=9.2 \mathrm{~cm}$.

Defining $\delta r_{i}(t)=r_{i}(t)-\left\langle r_{i}\right\rangle$, for each distance $\delta z$ between the thermometers, and each time lag $\tau$, we calculate the correlation by averaging $\delta r_{1}(t+\tau) \delta r_{2}(t)$ first within each block, then between the blocks. We then normalize this average by the product of the rms fluctuations $\sigma_{i}=\sqrt{\left\langle\delta r_{i}^{2}\right\rangle}$ :

$$
C(\delta z, \tau)=\frac{\left\langle\delta r_{1}(t+\tau) \delta r_{2}(t)\right\rangle}{\sigma_{1} \sigma_{2}}
$$

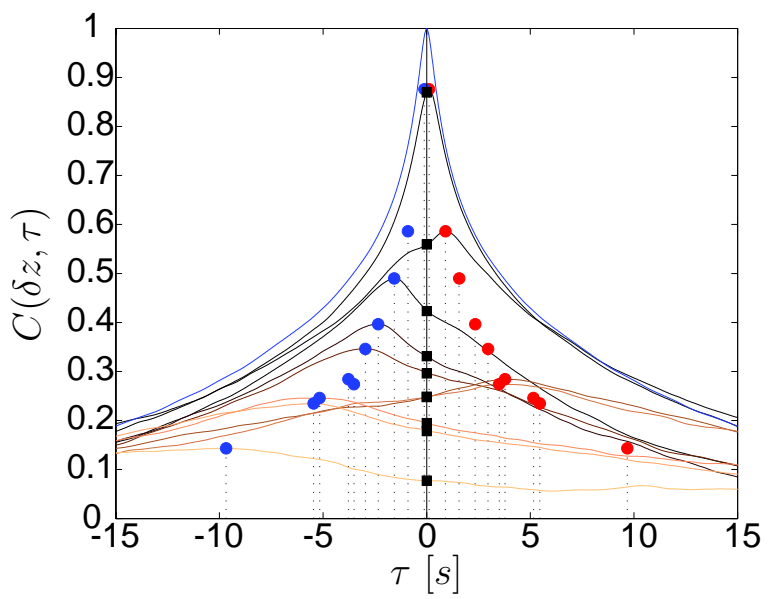

FIG. 3: The correlation function $C(\delta z, \tau)$ for various $\delta z$ in the high power case $(235 \mathrm{~W})$. The dots correspond to the position of the highest peak and its symetric compared to $\tau=0$. The squares mark the value of $C(\delta z, 0)$ for the different $\delta z$, from the darker to the lighter: $4 \mathrm{~mm}, 10 \mathrm{~mm}, 20 \mathrm{~mm}, 30 \mathrm{~mm}, 40 \mathrm{~mm}$, $50 \mathrm{~mm}$ (twice), $60 \mathrm{~mm}, 70 \mathrm{~mm}$, and $100 \mathrm{~mm}$. The blue (top) curve corresponds to the autocorrelation of one sensor (i.e. $\delta z=0)$.
Figure 3 presents the correlation function $C(\delta z, \tau)$ for various $\delta z$ ranging from $4 \mathrm{~mm}$ to $10 \mathrm{~cm}$, in the high power case. These functions can be seen as the sum of two components, of different amplitudes, but symetrically shifted from the origin. To stress this point, we systematically signal the position of the most visible "peak", and its symetric. Several remarks must be made:

- Each curve presents two peaks. These peaks indicate a delay between the two signals, the temperature perturbations traveling from one thermometer to the other.

- The presence of two peaks, approximately at symetric times $\pm \tau_{p}$, indicates that the flow undergoes reversals. They will be discussed in section $\mathrm{V}$.

- From the smallest distance curves, a correlation time can be extracted. It is in very good agreement with $\tau_{o}$ obtained from the spectrum (section IV, figure 5) of the bridge output fluctuations [12].

We now turn to the correlation length $\ell$. Strictly speaking, its definition assumes an exponential decay for $C(\delta z, 0)$ versus $\delta z(C(\delta z, 0)=\exp -(\delta z / \ell))$. Figure 4 shows these decays for both cases, the high and the low power. Both decays seem smoother than exponential, while an exponential approximation seems to be reasonable.

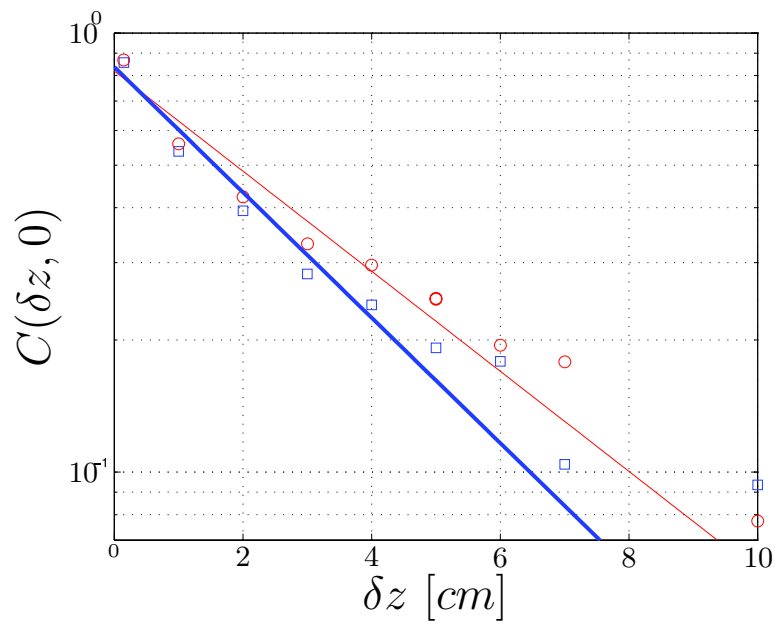

FIG. 4: The correlation function $C(\delta z, 0)$ versus $\delta z$. The lines are fits with an exponential decay.

The best fit gives $\ell=3.8 \mathrm{~cm}$ for the high power case $\left(L_{o}=11.4 \mathrm{~cm}\right)$, and $\ell=3 \mathrm{~cm}$ for the low power case $\left(L_{o}=9.2 \mathrm{~cm}\right)$. Thus, in any case, $\ell$ is smaller than the height of the bridge. This confirms our first hypothesis, that the rms fluctuations of the bridge are temperature rms fluctuations and not gradient ones. Obviously, a full confirmation needs a more complete study, but the evolution of $\ell$ seems proportional to that of $L_{o}$. 
To verify our second hypothesis, that is $a$ is constant in Eqs. 4,5, we have to come back on the velocity. From the time $\tau_{p}$ of occurence of the peaks, we can obtain a velocity $V_{p}=\delta z / \tau_{p}$. This velocity is independent of $\delta z$ within $\pm 5 \%$. For the high power $V_{p}=1.3 \mathrm{cms}^{-1}$, while for the low power $V_{p}=0.6 \mathrm{cms}^{-1}$. These values are much larger than $L_{o} / 2 \tau_{o}$ which was the estimation of the velocity in [12]. As we shall see in section $\mathrm{V}, V_{p}$ is however close to the rms velocity mesured using PIV in the present work. We thus must admit an error in [12], probably due to a bad calibration of distances in our first PIV measurement. We note that taking $a=1$, then $V_{\theta}=L / \tau_{o}=L_{o} / \tau_{o}$ is very close to $V_{p}$ and to the rms velocity. Indeed, taking $a=1$ gives for the high power $V_{\theta}=1.23 \mathrm{cms}^{-1}$, and for the low power $V_{\theta}=0.62 \mathrm{cms}^{-1}$.

From now on, we shall thus take $a=1$, and we define:

$$
L=L_{o}=\frac{\theta}{\beta}
$$

We can remark here that, contrary to our intuition, $\ell / \tau_{o}$ is much smaller than the rms velocity, while $V_{p}$ is close to it. This suggest a peculiar organisation for the coherent structures carrying the heat. Their travelling velocity as a whole is much smaller than the velocities occuring within the structure. This would be the case for a vortex, for instance, which generate velocities much larger than its own core velocity. However, the informations we have on this organisation are not sufficient to go further.

To resume the results of this section, the vertical temperature correlation length $\ell$ is smaller than the height $h$ of our bridge. We thus confirm that the rms output of the bridge $\theta$ must be interpreted as a temperature fluctuation amplitude, and not a gradient fluctuation amplitude. $L=\theta / \beta$ is then the intrinsic characteristic length we use in the definition of $R a$ and $R e$. It is much larger than the correlation length $\ell$, suggesting a subtle organisation of the flow.

\section{NUSSELT AND REYNOLDS NUMBERS}

We shall systematically use the length $L$ defined in Eq. 8 for the determination of $R a$ and $R e$, Eq. 3 and 6 . Then, it is worth discussing the real meaning of $\theta$. The output of the Wheatstone bridge, through the sensitivity factor of $2 \mathrm{mV} / \mathrm{K}$, measures:

$$
\frac{1}{2}\left(\left(T_{3}+T_{4}\right)-\left(T_{1}+T_{2}\right)\right)=T_{34}-T_{12}
$$

where 1 and 2 refer to the resistances at the top of the "I" structure (see figure 2b), 3 and 4 to those at the bottom. We just saw in the previous section that $(1,2)$ are decorrelated from $(3,4)$, their vertical distance $h(10 \mathrm{~cm}$ for the first cell, $5 \mathrm{~cm}$ for the second one) being larger than the correlation length.

At this point, it is useful to briefly discuss the structure of the flow and the temperature field, which will be detailled in section V. A large scale flow is present in the channel, ascending on one side and descending on the other, with occasional reversals, already mentioned. This flow creates a temperature difference between both sides of the "I" structure, at the same level, this difference, of absolute value $2 \Delta$, changing its sign at each reversal. Indeed, the rising flow, coming from the hot chamber, is slightly warmer $(+\Delta)$ than the sinking flow, coming from the cold chamber $(-\Delta)$. The time between these reversals can be long, 10 minutes or more. As a result, $T_{1}$ and $T_{2}$ are correlated, the fluctuations of $T_{12}=\left(T_{1}+T_{2}\right) / 2$ being poorly sensitive to the reversals dynamics. The mean squared deviation of the bridge output then gives:

$$
\begin{aligned}
\theta^{2} & =\left\langle\delta T_{34}^{2}\right\rangle+\left\langle\delta T_{12}^{2}\right\rangle \\
& =2\left\langle\delta T_{12}^{2}\right\rangle
\end{aligned}
$$

$\theta$ is thus different from the local rms temperature fluctuations, due to the reversals. The frequency spectrum of the bridge output is also different in shape from the local temperature one, particularly in its low frequency part, sensitive to the reversals dynamics.

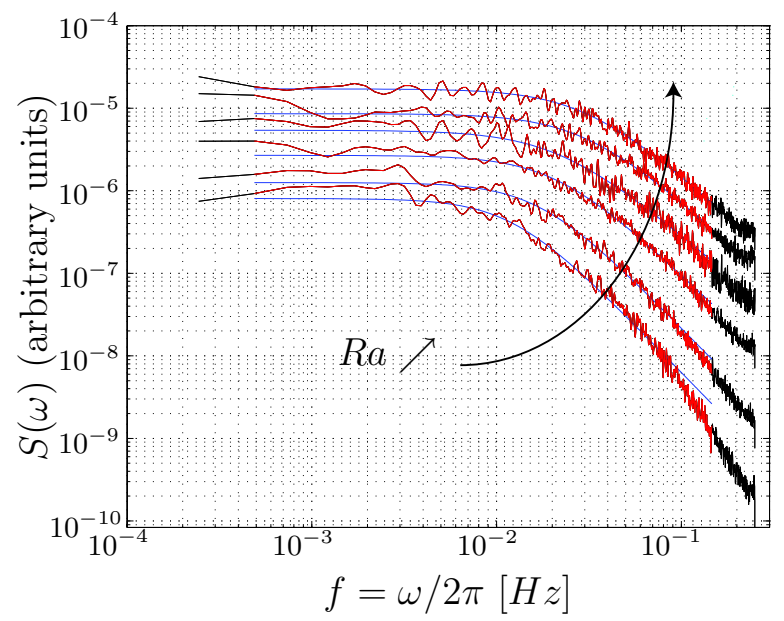

FIG. 5: The spectra of the bridge output, for various $R a$ values (second cell, $P r=6$ ). The lines correspond to the fit used for extracting $\tau_{o}$. The range for the fit is signaled in red on the spectrum.

We recall that this spectrum gives us the time $\tau_{o}$, from which we obtain the Reynolds number through Eq. 6 . Figure 5 shows a series of such spectra, corresponding to various Rayleigh numbers.

In order to improve the precision on $\tau_{o}$ we used a fit of these spectra $S(\omega)$. Indeed, considering $\omega S(\omega)$ gives a maximum in the neighborhood of $\omega_{o}=1 / \tau_{o}$. We thus fit $\omega S(\omega)$ with the function:

$$
S_{o} \frac{\omega \tau_{\gamma}}{1+\left(\omega \tau_{\gamma}\right)^{\gamma}}
$$

where $S_{o}, \tau_{\gamma}$, and $\gamma$ are adjustable parameters. In fact, the optimum $\gamma$ has very little variations, close to 1.8 for 
the first cell, and 2 for the second one. For each cell, we thus used the same $\gamma$ for all spectra. We define the characteristic time $\tau_{o}$ as:

$$
\tau_{o}=(\gamma-1)^{1 / \gamma} \tau_{\gamma}
$$

In this way, two fits having their maximum at the same frequency give the same value for $\tau_{o}$, whatever $\gamma$ is.

In reference [12], we checked the vertical homogeneity of the gradient $\beta$ in the channel for the first cell. We made the same test in the second cell, measuring the gradient at various heights. Figure 6 gives the results, for a driving power of $80 \mathrm{~W}$, and a mean temperature of $25^{\circ} \mathrm{C}$.

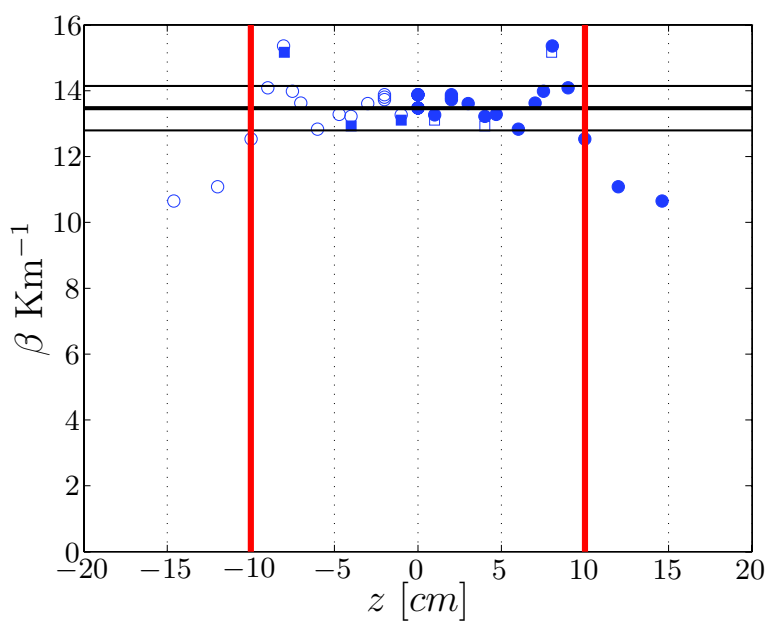

FIG. 6: Temperature gradient versus the height in the cell, for the second cell. Only full symbols correspond to real measurements. Open symbols are simply symetric to the full ones compared to the middle of the cell. The two vertical bars signal the ends of the channel. The thick horizontal line stands for the average of $\beta(z)$ in the center of the channel. The lower and upper horizontal lines correspond to $\pm 5 \%$ of this mean value.

Positions between $-10 \mathrm{~cm}$ and $10 \mathrm{~cm}$ correspond to the center of the bridge being in the channel. Between $-20 \mathrm{~cm}$ and $-10 \mathrm{~cm}$ it is in the hot chamber, and in the cold one between $10 \mathrm{~cm}$ and $20 \mathrm{~cm}$. Compared to the first cell [12], the conical shape of the chambers seems to result in a significant vertical gradient in these chambers. Correlatively, the channel end effect is strongly reduced, and the gradient can be considered as constant on a large range around the middle of the cell. Note also the large value of the gradient here $\left(\beta=13.5 \mathrm{Km}^{-1}\right)$. For the same power $(80 \mathrm{~W})$ and the same temperature $\left(25^{\circ} \mathrm{C}\right)$, the gradient is $\beta=1.9 \mathrm{Km}^{-1}$ in the first cell. This measurement confirms that in the central part of the channel, the temperature gradient $\beta$ is constant within less than $\pm 5 \%$.

We now come to the presentation of our results, for $\mathrm{Nu}$ and $R e P r$ versus $R a P r$. The various symbols on figure 7 correspond to different mean temperatures, and thus different Prandtl numbers $\operatorname{Pr}: 20^{\circ} \mathrm{C}, \operatorname{Pr}=6.6 ; 30^{\circ} \mathrm{C}$,
$\operatorname{Pr}=5.3 ; 40^{\circ} \mathrm{C}, \operatorname{Pr}=4.1$. The lower $\operatorname{RaPr}$ (diamonds) correspond to the second cell and a mean temperature of $25^{\circ} \mathrm{C}(\operatorname{Pr}=6)$. The error bars on $N u$ are based on an estimated uncertainty of $0.5 \mathrm{~W}$ on the heat leaks, and $210^{-3}{ }^{\circ} \mathrm{C}$ uncertainty on the temperature difference measured by the bridge. The latter uncertainty also gives that of $\beta$ which, together with $2 \%$ on $\theta$ yield the error bars on $L$ and $R a$ (see figure 8).

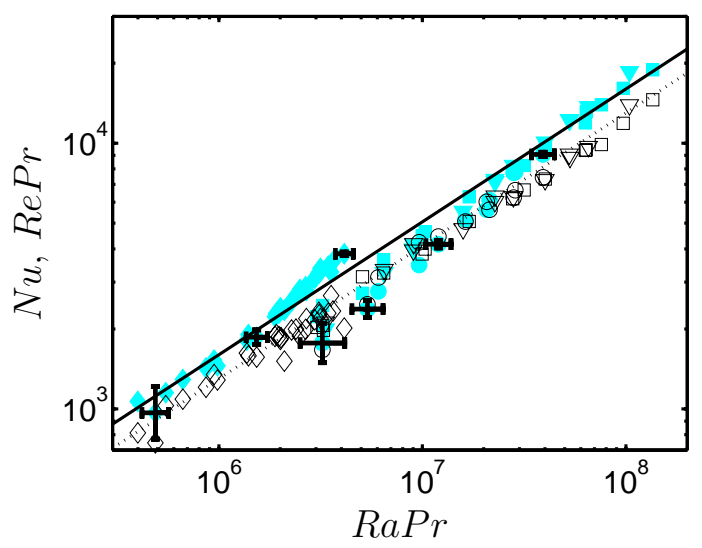

FIG. 7: Evolution of $N u$ (full symbols) and $\operatorname{Re} \operatorname{Pr}$ (open symbols) versus $R a P r$. First cell: $\circ, \operatorname{Pr}=6.6 ; \nabla, \operatorname{Pr}=5.3 ; \square$, $\operatorname{Pr}=4$.1. Second cell: $\diamond, \operatorname{Pr}=6$. Full line, proposed average law for $N u$ results: $N u=1.6 \sqrt{R a P r}$. Dotted line, proposed average law for $\operatorname{RePr}$ results: $\operatorname{Re} P r=1.3 \sqrt{\operatorname{RaPr}}$

We note the good agreement for the Re results between the two cells, and for both cells, with a law:

$$
R e P r \simeq 1.3 \sqrt{R a P r}
$$

We remark that this agreement does not depend on the choosen value for $L$, due to our definition of $R e$, Eq. 6 . The above law indicates that the velocity does not depend on the diffusivity $\kappa$, but depends on the viscosity $\nu$ through the dependence of $L$ versus $R e$ (see below, figure 8).

As for the heat transport, despite the large differences noted above for temperature gradients between the two cells, they agree when normalized as $N u$. A common law could be:

$$
N u \simeq 1.6 \sqrt{\operatorname{RaPr}}
$$

Note however that the tendency within each cell, is to a steeper logarithmic slope, but the difference with $1 / 2$ is close to the error bar. Here, the agreement between cells strongly depends on the choice of $L$. We used the same rule, Eq. 8, for its determination in the second cell. It gives a roughly constant value, close to $d$. Figure 8 resumes the variations of $L / d$ for both cells versus Re, showing the agreement between them. It also confirms the existence of the threshold $R e * \simeq 600$. For $R e<R e *$, 


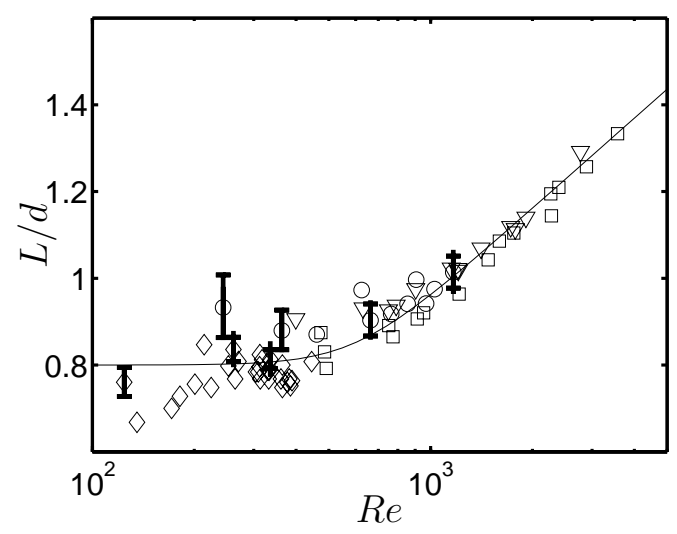

FIG. 8: Dependence of $L / d$ versus Re for both cells. Same symbols as in figure 7 . The line correspond to a fit of our data with the formula: $(L / d)=0.075 \ln \left(1+(R e / R e *)^{4}\right)+0.8$, with Re* $=600$.

$L / d$ is constant, For $R e>R e *, L / d$ increases with $R e$, approximately linear in $\ln R e$.

Can we compare with the results of the numerical simulation of reference [10]? The difficulty is in the choice of the characteristic length. In reference [10], periodic boundary conditions are used, and the characteristic length is the period of the system. In our case, we shall see in section VI that a half sine period can fit the vertical velocity $x$-profile, the period being $18 \mathrm{~cm}$, i.e. approximately $2 d$. For a valuable comparison, their $R a$ values should be divided by $2^{4}=16$ and their $R e$ values by 2 . Then, the laws they propose, $N u \simeq 0.7 \sqrt{R a}$ and $R e \simeq 1.1 \sqrt{R a}(\operatorname{Pr}=1)$ must be read:

$$
N u \simeq 2.8 \sqrt{R a} \quad ; \quad R e \simeq 2.2 \sqrt{R a}
$$

Considering the uncertainty on this conversion factor, the agreement is not bad. In particular, the ratio $R e / N u$ agrees well. There remains a difference of almost a factor 2 on both $N u$ and $R e$. Is it due to their absence of confinement in $x$ which favors velocity fluctuations, or to the periodicity in $z$ which prevents the free formation of plumes? The answer matters if we have to correctly model free convection in stars or atmospheres, but it will need further studies.

We can also compare with the results of reference [11]. They look at the transient free convection in a vertical tube connecting two chambers with different salt concentrations. Thus, they have a flux of salt instead of a flux of heat, and the salt diffusion coefficient (resp. the Schmidt number $S c$ ) takes the place of $\kappa($ resp. $P r)$. They obtain:

$$
N u \simeq 0.88 \sqrt{R a S c} \quad ; \quad R e S c \simeq \sqrt{R a S c}
$$

The agreement with our results is good if we consider that they take $d$ as the characteristic length scale, which is slightly larger than our $L$ (their Reynolds number is small, lower than the threshold Re*). Moreover, they do not measure directly the density gradient in the tube, but estimate it from the difference in salt concentration between the chambers.

To resume the results of this section, the $N u$ and $R e$ dependences versus $R a$ and $P r$ are coherent with a purely inertial behaviour, if $L=\theta / \beta$ is the characteristic length entering the definition of $R a$ and $R e$. It means that the heat flux and the fluid velocity do not depend on the heat molecular diffusivity, and that their dependence on the kinematic viscosity is entirely contained in the Re dependence of $L$. This dependence changes at some threshold $R e * \simeq 600$. For $R e<R e *, L$ is constant and close to $d$. Previous equivalent studies $[10,11]$ fit into this regime and we reasonably agree with them. For $R e>R e *, L$ grows, approximately linear in $\ln R e$. Can we extrapolate this dependence up to the largest $R e$, or do some saturation occurs? We presently do not know.

\section{DESCRIPTION OF THE VELOCITY FIELD}

In this section, we describe the velocity field as obtained through our PIV system [18]. As visualising the cell prevents us to use the thermal screen, the average fluid temperature is choosen to be that of the room $\left(30^{\circ} \mathrm{C}\right)$, and only the bottom plate is thermally coated. Most of the measurements have been made with the first cell. The second one has been used for checking some of the conclusions.

As seen in [12], the channel ends effects influence the flow a few $\mathrm{cm}$ from these ends. It is why we limit our study in the first cell to the range $-5 \mathrm{~cm}<z<5 \mathrm{~cm}$. As we shall see later, even in this restricted range, ends have a visible, but negligible, effect.

Two different input powers have been explored, $180 \mathrm{~W}$ $\left(R a=1.51 \times 10^{7}\right)$ and $93 \mathrm{~W}\left(R a=7.9 \times 10^{6}\right)$. The discussion below will mainly concern the lowest. Figure 9 shows typical instantaneous maps of the two components, $v_{x}$ and $v_{z}$.

In most of the pictures, for this first cell, the flow is globally ascending in the left hand part, and descending in the right one (as in figure 9, top), or the opposite (figure 9 , bottom). These flows, we call $\Phi$-flows. Some of the pictures show a flow globally ascending in both parts (remember that we record only a sheet of the flow), or descending. We call this kind of flow $\Xi$-flows. $\Phi$-flows have a typical mixing layer structure. In order to discuss them, we have first to extract the average profile of the velocity field.

With this in mind, we first define $\epsilon_{l}$, which is 1 if the average of the vertical velocity in the left part is positive, and $\epsilon_{l}=-1$ if it is negative. We also define $\epsilon_{r}$ which is -1 if the average of the vertical velocity in the right part is positive, and $\epsilon_{r}=1$ if this latter average is negative. For $\Phi$-flows, $\epsilon_{l}=\epsilon_{r}$. Reciprocally, for $\Xi$-flows, $\epsilon_{l}=-\epsilon_{r}$. 

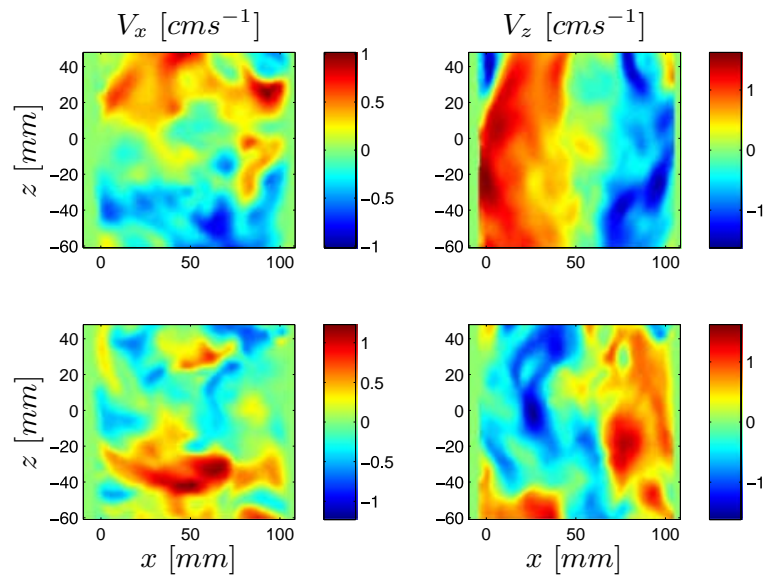

FIG. 9: Two typical instantaneous maps of the two velocity components algebraic values. Note that the color code is not the same for $v_{x}$ and $v_{z}$. Top: $\epsilon=+1$; Bottom: $\epsilon=-1$ (see text).

We then define:

$$
\epsilon=\frac{1}{2}\left(\epsilon_{l}+\epsilon_{r}\right)
$$

which is zero for $\Xi$-flows, and \pm 1 for $\Phi$-flows.

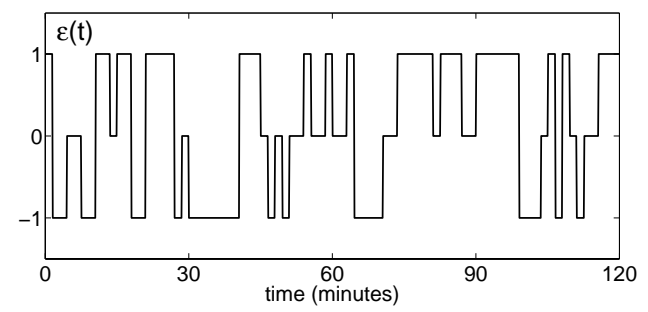

FIG. 10: Schematic dynamics of the reversals.

Figure 10 shows $\epsilon(t)$ for a 2 hours period record at $93 \mathrm{~W}$. As explained in section II, these records consist of 80 blocks of 19 instantaneous velocity maps, the duration of a block being 1s. The interval between blocks is thus 89s. Clearly, this record shows no characteristic time. The dynamics of these reversals will be studied in another paper.

Figure 11 shows the average fields $\left\langle\epsilon v_{x}\right\rangle$ and $\left\langle\epsilon v_{z}\right\rangle$ where \langle\rangle stands for time average divided by the time average of $\epsilon^{2}$, to take into account only the $\Phi$ flows. $\left\langle\epsilon v_{z}\right\rangle$ presents a reasonable invariance versus $z$, as expected for the central part of the channel. In contrast $\left\langle\epsilon v_{x}\right\rangle$ shows unexpected non-zero average at top and bottom parts, as if a roll was superimposed to the expected parallel flow. Note however that the amplitude of this roll is much weaker than the parallel component. Moreover, a comparison with the velocity field in the second cell shows that these finite average horizontal velocity spots follow the channel ends when the relative distance between them increases. We can thus consider them as a trace of end effects.
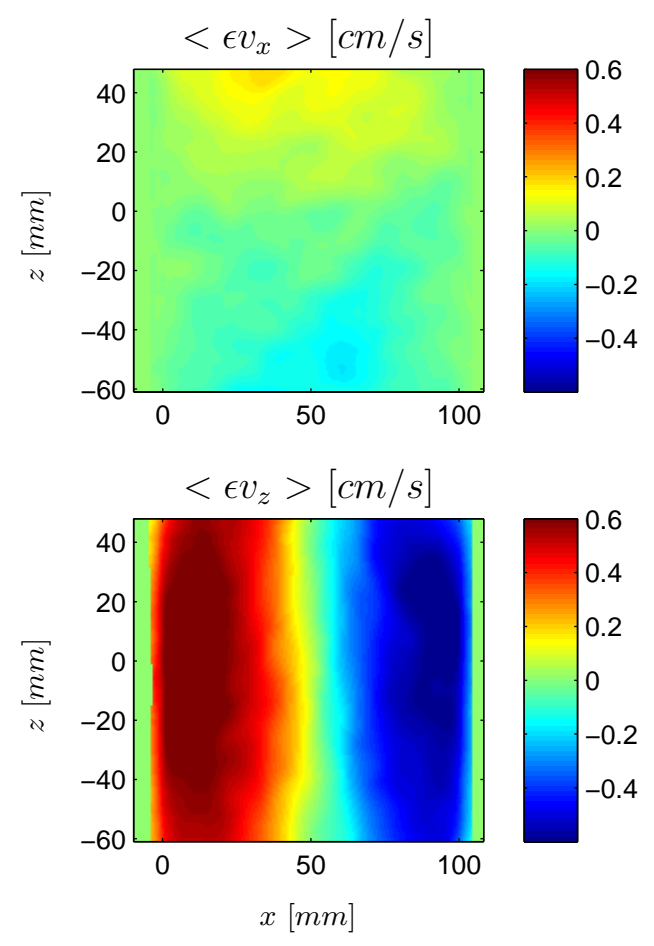

FIG. 11: Time averages of $\epsilon v_{x}$ and $\epsilon v_{z}$.

Due to the observed translational invariance, it is reasonable to define the $z$-average:

$$
U_{z}(x)={\overline{\left\langle\epsilon v_{z}\right.}}^{z}
$$

as the profile of the vertical velocity. Here $\overline{\langle.\rangle}^{z}$ stands for a time average, itself averaged along $z$.

As for the $x$ component, let us define:

$$
U_{x}(x)=\sqrt{{\overline{\left(\left\langle\epsilon v_{x}\right\rangle\right)^{2}}}^{z}}
$$

which will be useful when looking at fluctuations, in the next section. Moreover, comparing them on the same graph, figure 12 allows to estimate the relative importance of this artefact.

To resume the results of this section, apart from erratic reversals, the mean flow organizes in two collumns, of rising hot fluid and sinking cold fluid. The non zero horizontal velocity averages clearly identify as end effects.

\section{VELOCITY AND TEMPERATURE FIELDS: LOCAL BUDGETS}

The study of the velocity field we have made allows more than determining an average profile. It allows to 


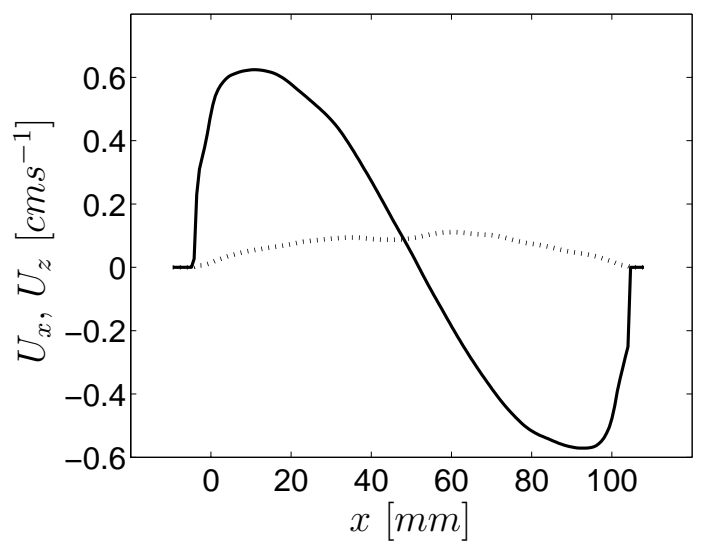

FIG. 12: $U_{z}$ (full line) and $U_{x}$ (dotted line) profiles. See the text for the definition of these $z$ and time averages.

look at the Reynolds stresses induced by the fluctuations, which, in turn, give indirect information about the horizontal temperature profile:

$$
\Theta(x)=\overline{\langle\epsilon T\rangle}^{z}
$$

Indeed, the momentum budget along the vertical direction is, within the Boussinesq approximation:

$$
\partial_{t} v_{z}+\partial_{j}\left(v_{z} v_{j}+\frac{p}{\rho} \delta_{z, j}-\nu \partial_{j} v_{z}\right)=g \alpha T
$$

where repeated index $j$ means summation on $j=x, y, z$, and $\delta_{z, j}$ is the Kronecker symbol. To be specific, $p$ is the effective pressure, including the potential term $\rho g z . \rho$ is the fluid density under this pressure and a temperature equal to the time averaged and horizontally averaged one at $z=0$. Multiplying Eq. 21 by $\epsilon$, and averaging over $t$ and $z$ gives:

$$
\partial_{x}\left({\overline{\left\langle\epsilon v_{x} v_{z}\right\rangle}}^{z}-\nu \partial_{x} U_{z}\right)=g \alpha \Theta
$$

Indeed, to obtain Eq. 22 we also average over $y$, the third dimension. In the experimental PIV study, we had only access to a sheet, and we assumed the statistical invariance along $y$. From now on, we shall consider that the subscript $₹$ systematically implies an average on $y$.

Eq. 22 alone shows the importance of $\tau_{x z}={\overline{\left\langle\epsilon v_{x} v_{z}\right\rangle}}^{z}$ which represents the momentum flux along $x$ when the viscosity can be neglected. Figure 13 shows the $x$ variations of $\tau_{x z}$, together with the two other significant Reynolds stresses: $\tau_{z z}={\overline{\left\langle\left(\epsilon v_{z}-\left\langle\epsilon v_{z}\right\rangle\right)^{2}\right\rangle}}^{z}$ and $\tau_{x x}=$ ${\overline{\left\langle\left(\epsilon v_{x}-\left\langle\epsilon v_{x}\right\rangle\right)^{2}\right\rangle}}^{z}$. Indeed, the last two play little role as stresses, but give a good estimate of the amplitude of the fluctuations.

Several remarks can be made. First, $\tau_{x z}$ is much larger than the viscous stress $\left|\nu \partial_{x} U_{z}\right|\left(2.510^{-3} \mathrm{~cm}^{2} \mathrm{~s}^{-2}\right.$ in the center), except very close to the walls (where anyway the precision of our PIV is doubtful). It means that most of

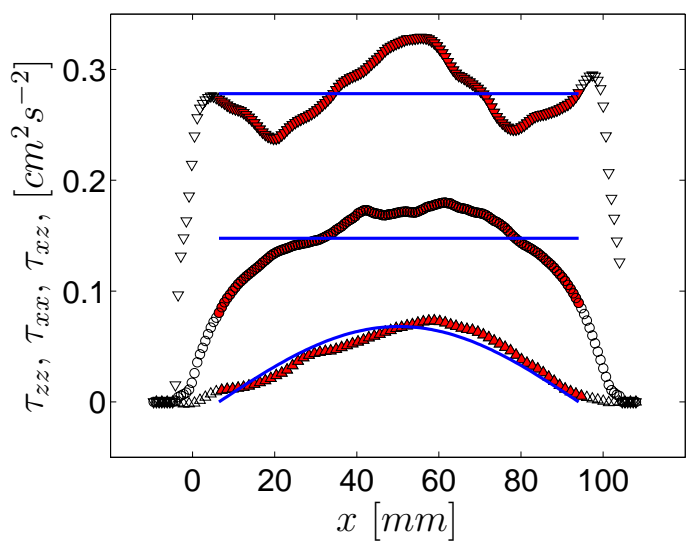

FIG. 13: The Reynolds stresses $\tau_{x x}(\circ), \tau_{z z}(\nabla)$, and $\tau_{x z}$ $(\triangle)$, versus $x$, for the lowest power $(93 \mathrm{~W})$. The full symbols correspond to the range of the fit $\left(x_{-}=7 \mathrm{~mm}, x_{+}=94 \mathrm{~mm}\right)$. The lines show the proposed fit.

the momentum created by the buoyancy forces directly exchange from one side of the channel to the other, with almost nothing going to the walls. We can practically neglect the friction on the walls.

Second, $\tau_{x x}$ and $\tau_{z z}$ are almost constant across the channel, suggesting a kind of homogeneity in the "turbulent fluid" which we study. However, $\tau_{z z}>\tau_{x x}$. This "turbulent fluid" is anisotropic. Finally, note that $\tau_{z z}$ is of the order of $U_{z}^{2}$. The vertical velocity fluctuations are large.

We can also write the enthalpy budget in the channel:

$$
C_{p} \partial_{t} T+\partial_{j}\left(q_{j}+v_{j} C_{p} T\right)=0
$$

where $C_{p}$ is the constant pressure heat capacity per unit volume, and $\vec{q}$ is the diffusive heat flux. Writing:

$$
\vec{Q}=\vec{q}+C_{p} T \vec{v}
$$

the total heat flux, diffusive plus convective, we note that $Q_{x}$ changes its sign at each reversal, on average, while $Q_{z}$ does not. Then, multiplying Eq. 23 by $\epsilon$, and averaging on $t$ and $z$ gives:

$$
C_{p} \beta U_{z}=\partial_{x}{\overline{\left\langle\epsilon Q_{x}\right\rangle}}^{z}
$$

giving indirect access to the horizontal heat flux $\overline{\left\langle\epsilon Q_{x}\right\rangle}$. To obtain this result, it is convenient to define $\overrightarrow{v^{\prime}}$ and $T^{\prime}$ such that:

$$
\begin{aligned}
\epsilon(T+\beta z) & =\Theta(x)+T^{\prime} \\
\epsilon v_{x} & =v_{x}^{\prime} \\
\epsilon v_{z} & =U_{z}(x)+v_{z}^{\prime}
\end{aligned}
$$

$\overrightarrow{v^{\prime}}$ and $T^{\prime}$ have zero average, and their statistics and correlation are independent of $z$. 
The relative constancy of $\tau_{x x}$ and $\tau_{z z}$ suggests, as discussed in the next section, to define effective transport coefficients for this quasi-homogeneous "turbulent fluid". For instance, we can define a "turbulent viscosity" through the relation:

$$
{\overline{\left\langle\epsilon v_{x} v_{z}\right\rangle}}^{z}=-\nu^{t u r b} \frac{d U_{z}}{d x}
$$

Having access, both, to the temperature profiles and to the heat fluxes in the vertical and horizontal directions, effective heat conductivities can be defined as follows:

$$
\begin{aligned}
{\overline{Q_{z}}}^{x}=Q & =\frac{C_{p}}{d} \int_{0}^{d} U_{z}(x) \Theta(x) d x+k_{z}^{\text {turb }} \beta \\
\overline{\left\langle\epsilon Q_{x}\right\rangle} & =-k_{x}^{\text {turb }} \frac{d \Theta}{d x}
\end{aligned}
$$

However, with the small statistics we have, and the corresponding noise on averaged quantities we cannot directly use Eqs. 27 to 29. It is why we smooth the obtained profiles through a simplified model.

We model the major central part of the flow, letting aside two regions $x<x_{-}$and $x_{+}<x$ as "boundary layers" close to the vertical walls. Considering that the momentum flux toward the walls is very small, a stress-free boundary flow well fits the profile of the vertical velocity:

$$
U_{z}(x)=U \cos \left(\pi \frac{x-x_{-}}{x_{+}-x_{-}}\right)
$$

We can then determine $\nu^{\text {turb }}$, using Eq. 27, and approximating $\tau_{x z}(x)$ with a sine function:

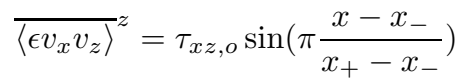

with:

$$
\tau_{x z, o}=\nu^{t u r b} \frac{\pi U}{\left(x_{+}-x_{-}\right)}
$$

Having $\nu^{\text {turb }}$, we can return to Eq. 22, and determine:

$$
\begin{aligned}
\Theta(x) & =\left(\nu^{\text {turb }}+\nu\right) \frac{\pi^{2} U}{g \alpha\left(x_{+}-x_{-}\right)^{2}} \cos \left(\pi \frac{x-x_{-}}{x_{+}-x_{-}}\right) \\
& =\Theta_{o} \cos \left(\pi \frac{x-x_{-}}{x_{+}-x_{-}}\right)
\end{aligned}
$$

Finally, using Eqs. 25, 29, we can determine $k_{x}^{\text {turb }}$ :

$$
k_{x}^{\text {turb }}=C_{p} \beta \frac{\left(x_{+}-x_{-}\right)^{4}}{\pi^{4}} \frac{g \alpha}{\nu^{\text {turb }}+\nu}
$$

The relative values of these effective transport coefficients, and their physical interpretation, will be discussed in the next section. Let us here briefly discuss the precision we can expect on them. We shall first consider the bounds of the fits, $x_{+}$and $x_{-}$, as given, and discuss later the influence of their choice.
The averages are obtained from 80 blocks of 20 frames. The simple fact that the sign of $\epsilon$ is clear on each block shows that the error bar on each quadratic quantity obtained from a single block is smaller than its mean value. We estimate this error bar to be half the mean value. Averaging on 80 blocks thus yield to $6 \%$ on each quadratic quantity, and $3 \%$ on their square root. The precision on $\beta$ is estimated at $2 \%$. All these estimates obviously hold for reasonably high input power, where heat leaks are negligible.

The precision on $\nu^{\text {turb }}$ is limited by the precision on $U$ and that on $\tau_{x z}$. Following the above discussion it should be $7 \%$. The precision on $\Theta_{o}$ is simply limited by the precision on $\tau_{x z}$, that is $6 \%$. The precision on $k_{x}^{\text {turb }}$ depends on $\beta$ and $\nu^{\text {turb }}$. We thus estimate it as $7 \%$.

As $\nu^{\text {turb }}$ is much larger than $\nu$, and considering its precision, $\nu$ will always be negligible compared to $\nu^{\text {turb }}$. Another quantity of interest will be the turbulent Prandtl number:

$$
P r^{t u r b}=\frac{C_{p} \nu^{t u r b}}{k_{x}^{t u r b}}
$$

The above discussion yield to $10 \%$ precision on $\operatorname{Pr}^{\text {turb }}$.

Let us now discuss on the influence of $x_{+}$and $x_{-}$. Indeed, we remarked that their choice have very little influence on the calculated value of $\nu^{\text {turb }}$. This was to be expected, as $\nu^{\text {turb }}$ is obtained from the comparison of two really measured quantities, $U_{z}(x)$ and $\tau_{x z}(x)={\overline{\left\langle\epsilon v_{x} v_{z}\right.}}^{z}$. The values proposed for $\nu^{\text {turb }}$ should thus be reliable, within their estimated uncertainty. The consequence, however, is that $k_{x}^{\text {turb }}$ behaves as $\left(x_{+}-x_{-}\right)^{4}$ (see Eq. 34).

We thus proceed as follows. We define symetrized versions of the data, namely:

$$
U_{\text {sym }}(x)=\left(U_{z}(x)-U_{z}(d-x)\right) / 2
$$

and

$$
\tau_{\text {sym }}(x)=\left(\tau_{x z}(x)+\tau_{x z}(d-x)\right) / 2
$$

and we call $d U$ and $d \tau$ the root mean squared differences between these symetrized data and the sine approximations. We then search for the minimum of $\left(d \tau / \tau_{x z, o}\right)^{2}+4(d U / U)^{2}$ versus $\left(x_{+}-x_{-}\right)$. This minimum, mainly dominated by $d \tau$, is sufficiently peaked as to consider $\left(x_{+}-x_{-}\right)$as determined within less than $2 \%$.

In this section, we showed that the mean flow is coherent with the most simple model, implying constant effective diffusivities and stress free boundary conditions. Measuring the Reynolds stresses give us access to the horizontal temperature profile and to the horizontal corresponding heat flux. Concrete values, and an interpretation of the measured turbulent diffusivities, are given in the next section 


\section{MIXING LENGTHS AND PLUMES}

In this section, we examine the pertinence of effective transport coefficients to describe our "turbulent fluid". Having in mind a comparison with the kinetic theory of gases, a transport coefficient like $\nu^{\text {turb }}$ can be seen as the product of the characteristic velocity of fluid particles (the momentum carriers) with a characteristic length, the Prandtl's "mixing length". As the corresponding transport is along the $x$ axis, we can take $\sqrt{\tau_{x x}}$ as the characteristic velocity, and write:

$$
\nu^{t u r b}=L_{\nu} \sqrt{\tau_{x x}}
$$

Such a description makes sense only if $L_{\nu}$ is smaller than the characteristic length of the mean flow, of order $d$. In the same spirit, we can write:

$$
k_{x}^{t u r b}=C_{p} L_{k x} \sqrt{\tau_{x x}}
$$

and:

$$
k_{z}^{t u r b}=C_{p} L_{Q} \sqrt{\tau_{z z}}
$$

The following table compares two experiments at the average temperature of $30^{\circ} \mathrm{C}$, with the total applied power of $93 \mathrm{~W}$ and $180 \mathrm{~W}$, in the first cell. $\Delta X / X_{m}$ refers to the difference between the considered quantity values in both cases, divided by their mean value. $V_{\theta}=L / \tau_{o}$.

\begin{tabular}{||l||c|c|c||}
\hline Quantity X & $93 \mathrm{~W}$ & $180 \mathrm{~W}$ & $\Delta X / X_{m}$ \\
\hline \hline$P r=\nu / \kappa$ & 5.22 & 5.12 & $-2 \%$ \\
\hline$\beta\left[\mathrm{K} \cdot \mathrm{m}^{-1}\right]$ & 1.82 & 2.39 & $27 \%$ \\
\hline$L[\mathrm{~cm}]$ & 10.1 & 11.1 & $9 \%$ \\
\hline$V_{\theta}\left[\mathrm{m} \cdot \mathrm{s}^{-1}\right]$ & $8.9 \times 10^{-3}$ & $1.18 \times 10^{-2}$ & $9 \%$ \\
\hline \hline$N u$ & 8300 & 12200 & $38 \%$ \\
\hline$R a$ & $5.4 \times 10^{6}$ & $1.03 \times 10^{7}$ & $63 \%$ \\
\hline$R e$ & 1170 & 1730 & $39 \%$ \\
\hline \hline$x_{-}[\mathrm{mm}]$ & 7.1 & 5.4 & $-27 \%$ \\
\hline$x_{+}[\mathrm{mm}]$ & 93.4 & 94.9 & $2 \%$ \\
\hline$U\left[\mathrm{~m} \cdot \mathrm{s}^{-1}\right]$ & $6.2 \times 10^{-3}$ & $7.6 \times 10^{-3}$ & $20 \%$ \\
\hline$\tau_{z z}\left[\mathrm{~m}^{2} \cdot \mathrm{s}^{-2}\right]$ & $2.8 \times 10^{-5}$ & $4 \times 10^{-5}$ & $35 \%$ \\
\hline$\tau_{x x}\left[\mathrm{~m}^{2} \cdot \mathrm{s}^{-2}\right]$ & $1.5 \times 10^{-5}$ & $2.1 \times 10^{-5}$ & $33 \%$ \\
\hline$\tau_{x z, o}\left[\mathrm{~m}^{2} \cdot \mathrm{s}^{-2}\right]$ & $0.68 \times 10^{-5}$ & $1.0 \times 10^{-5}$ & $39 \%$ \\
\hline \hline$\Theta_{o}[\mathrm{~K}]$ & 0.079 & 0.112 & $35 \%$ \\
\hline$\nu^{\text {turb }}\left[\mathrm{m}^{2} \cdot \mathrm{s}^{-1}\right]$ & $3.00 \times 10^{-5}$ & $3.77 \times 10^{-5}$ & $23 \%$ \\
\hline$k_{x}^{\text {turb }} / C_{p}\left[\mathrm{~m}^{2} \cdot \mathrm{s}^{-1}\right]$ & $1.1 \times 10^{-4}$ & $1.3 \times 10^{-4}$ & $18 \%$ \\
\hline$P r^{\text {turb }}$ & 0.28 & 0.29 & $4 \%$ \\
\hline$k_{z}^{\text {turb }} / C_{p}\left[\mathrm{~m}^{2} \cdot \mathrm{s}^{-1}\right]$ & $0.93 \times 10^{-3}$ & $1.38 \times 10^{-3}$ & $39 \%$ \\
\hline \hline$L_{\nu}[\mathrm{cm}]$ & 0.78 & 0.83 & $6 \%$ \\
\hline$L_{k x}[\mathrm{~cm}]$ & 2.8 & 2.9 & $2 \%$ \\
\hline$L_{Q}[\mathrm{~cm}]$ & 17.6 & 21.8 & $21 \%$ \\
\hline \hline$T_{a}$ & & & \\
\hline
\end{tabular}

Table I: Summary of the various quantities directly measured, or derived from the measurements for two experiments at the average temperature of $30^{\circ} \mathrm{C}$, in the first cell. The last column allows to distinguish between the approximately constant quantities and the varying ones.

Let us comment first on the values of $x_{+}$and $x_{-}$. They are close to the walls, the closer when the Reynolds number is higher. The relative size of the "boundary layer" is smaller when $R e$ is larger as one could have expected. Thus the process we followed to determine $x_{+}$and $x_{-}$ gives reasonable values.

Looking at the last column, we see that some quantities exhibit changes larger than the error bar, other not. Specifically, we note that $P r^{\text {turb }}$ is the same in both cases. Qualitatively, a value close to 1 had to be expected, as the carriers of both momentum and energy are the fluid particles themselves. The value obtained, $0.28 \pm 0.03$ differs sufficiently from 1 to be non trivial. For example, the Prandtl number of a monoatomic gas as Helium is $\operatorname{Pr}=0.7$.

The "mixing lengths" show contrasting behaviors. $L_{\nu}$ and $L_{k x}$ are short, much smaller than $d$, and can be considered as constant. They correspond to the expected properties of such quantities. It means that their values can be used for predicting the effective transport coefficients $\nu^{\text {turb }}$ and $k_{x}^{\text {turb }}$, and that these effective coefficients can yield to meaningful predictions, concerning the average flow.

Conversely, the values of $L_{Q}$ are not compatible with a mixing length approach. If we refer again to the kinetic theory of gases, these values suggest a "Knudsen" regime, where the mean free path of the carriers is larger than the size of the experiment. It is then more convenient to focus on the behavior of "individual carriers". This is such a model that we develop below.

In a very schematic point of view, let us then consider that heat is carried by intermittent coherent flows, which we call plumes. The velocity intensity of such a plume will be called $v$ and the temperature intensity $\vartheta$, in such a way that the instantaneous heat flux carried by the plume is:

$$
\mathcal{Q}=C_{p} v \vartheta / 2
$$

Now, let us call $\varpi$ the probability to be in a plume at a given point and time in the channel. The total heat flux will be:

$$
Q=\varpi \mathcal{Q}
$$

while the amplitude of the average flow $U$, and of the horizontal temperature profile $\Theta_{o}$ will be:

$$
U=\varpi v \quad ; \quad \Theta_{o}=\varpi \vartheta
$$

We can thus estimate $\varpi$, through:

$$
\varpi=\frac{C_{p} U \Theta_{o}}{2 Q}
$$

Both experiments presented in the above table give estimates of $\varpi$ in the neighborhood of $14 \%$. This ratio can 
also be seen as the part of the heat carried by the mean flow. In this respect, we can say that most of the heat is carried by plumes and not by the mean flow. Recent studies in Rayleigh-Bénard cells [8, 9] yield to the same conclusion.

\section{CONCLUSION}

In this paper, we have extended our previous measurements to another cell, of different size and aspect ratio, and found good agreement between the results of both cells. We also have studied the structure of the flow, both the mean flow and the fluctuations.

The temperature fluctuations, and their correlations, confirmed the hypothesis of short correlation length we made previouly. However, it also showed that the ratio between the correlation length $\ell$ and the correlation time $\tau_{o}$ is much smaller than the rms velocity. Certainly, further studies are necessary in order to confirm the impression we have that both have parallel behaviors versus $R a$ and $P r$. In any case, this only result shows that the organization of the coherent structures carrying the heat is very peculiar and has to be elucidated.
The mean flow appeared to be rather simple, if we disregard the reversals which will be the object of another work. The flow appears to be governed by effective horizontal transport coefficients $\nu^{\text {turb }}$ and $k_{x}^{\text {turb }}$, the corresponding "mixing lengths" being much smaller than the size of the channel. The effective "turbulent fluid" appears as homogeneous on roughly the whole width of the channel. It seems to be a scholar example for such concepts.

Conversely, the vertical heat transport cannot be modeled through an effective vertical heat conductivity. The good picture is more a transport by individual plumes. Again, further studies are needed to fully characterize these plumes.

\section{Acknowledgments}

We gratefully acknowledge for stimulating discussions with F. Toschi, D. Lohse, J.P. Hulin, F. Perrier, and P. Le Gal. We thank F. Dumas, M. Moulin and F. Vittoz for technical assistance. This work has been partly supported by the Agence Nationale pour la Recherche under the contract GIMIC BLAN07-3_184455.
[1] E. Siggia, "High Rayleigh number convection", Annu. Rev. Fluid Mech., 26, 137, (1994).

[2] X. Chavanne, F. Chillà, B. Chabaud, B. Castaing, B. Hébral, "Turbulent Rayleigh-Bénard convection in gaseous and liquid He" Phys. Fluids 13, 1300, (2001).

[3] S. Grossmann, D. Lohse, "Scaling in thermal convection: A unifying view ", J. Fluid Mech. 407 27-56 (2000); Grossmann S., Lohse D., "Fluctuations in RayleighBénard convection: The role of plumes.", Phys. Fluids 16 4462-4472 (2004).

[4] X. Chavanne, F. Chillà, B. Castaing, B. Hébral, B. Chabaud, and J. Chaussy, "Observation of the Ultimate Regime in Rayleigh-Bénard Convection", Phys. Rev. Lett. 79, 3648-3651 (1997).

[5] J. J. Niemela, L. Skrbek, K. R. Sreenivasan, R. J. Donnelly, "Turbulent convection at very high Rayleigh numbers" Nature, 404, 837-841, (2000).

[6] J. J. Niemela, K. R. Sreenivasan, "Confined turbulent convection", J. Fluid Mech. 481 355-384 (2003).

[7] R. H. Kraichnan, "Turbulent Thermal Convection at Arbitrary Prandtl Number", Phys. Fluids, 5, 1374-1389, (1962).

[8] X.D. Shang, X.L. Qiu, P. Tong and K.Q.Xia, "Measured local heat transport in turbulent Rayleigh-Bénard convection", Phys.Rev. Letters 90, 074501 (2003).

[9] Y. Gasteuil, W. Shew, M. Gibert, F. Chillà, B. Castaing, J. F. Pinton, "Lagrangian temperature, velocity and local heat flux measurements in Rayleigh Bénard Convection",
Phys.Rev. Letters 99, 234302, (2007).

[10] D. Lohse, F. Toschi, "Ultimate State of Thermal Convection", Phys. Rev. Letters 90, 034502 (2003); Calzavarini E., Lohse D., Toschi F., Tripiccione R., "Rayleigh and Prandtl number scaling in the bulk of Rayleigh-Bénard turbulence", Phys. Fluids 17, 055107, (2005).

[11] M.R. Cholemari, J.H. Arakeri, "Experiments and a model of turbulent exchange flow in a vertical pipe", International Journal of Heat and Mass Transfer, 48, 44674473 (2005).

[12] M. Gibert, H. Pabiou, F. Chillà, B. Castaing, "High Rayleigh number convection in a vertical channel", Phys.Rev. Letters 96, 084501, (2006).

[13] F. Perrier, P. Morat, J.L. Le Mouel, "Dynamics of Air Avalanches in the Access Pit of an Underground Quarry", Phys. Rev. Letters 89134501 (2002).

[14] PMMA: PolyMethylMethAcrylate.

[15] S. Ciliberto, S. Cioni, C. Laroche, "Large-scale flow properties of turbulent thermal convection", Phys. Rev. E 54 R5901 (1996)

[16] Typical values are $2 \mathrm{k} \Omega$ at $20^{\circ} \mathrm{C}$, and $1 \mathrm{k} \Omega$ at $40^{\circ} \mathrm{C}$.

[17] Typical values are $30 \Omega$ at $20^{\circ} \mathrm{C}$, with $0.5 \Omega / \mathrm{K}$ sensitivity, $0.4 \mathrm{~mm}$ in size.

[18] Davis-7, LaVision, GmbH.

[19] Sphericel 110P8, LaVision, GmbH.

[20] DPSS-Laser System, 532nm, Melles Griot.

[21] Imager Pro, LaVision, GmbH. 